\section{Lindenmeyer, $J$.}

\section{Offene Gruppen 1}

Weinheim, Beltz, 2010, 288 Seiten, 34,95 EUR

ISBN 3-621-27787-0

Mit ihrem Buch «Offene Gruppen 1» aus der Reihe «Therapie-Tools» liefern Johannes Lindenmeyer und 14 weitere Autoren eine Lösung für ein vor allem in der (teil-)stationären Versorgung häufiges Grundproblem. Einerseits basieren stationäre Psychotherapien in der Regel zu einem großen Teil auf Gruppenangeboten. Andererseits sind diese meist offen und die Patienten haben unterschiedliche Verweildauern. Diese Gründe machen die meisten Gruppentherapiemanuale, die für geschlossene, störungsspezifische Gruppen entwickelt wurden, nur bedingt nutzbar. Diesem Umstand trägt das hier vorgestellte Buch Rechnung, indem es eine große Anzahl an flexibel einsetzbaren Arbeitsblättern (ABs) zu den verschiedensten Themenbereichen zur Verfügung stellt.

In den ersten beiden Kapiteln erfolgt zunächst eine 20-seitige theoretische Einführung zu offenen Gruppen. Einzelund Gruppentherapie werden als sich ergänzende Behandlungsbausteine vorgestellt, wobei Vor- und Nachteile themenoffener bzw. themenzentrierter Gruppen sowie offener, halboffener bzw. geschlossener Gruppen dargestellt werden. Der Einsatz von ABs soll hierbei als Strukturierungs- und Orientierungshilfe dienen, zur Kommunikationserleichterung im Team, aber auch zur Herstellung von Transparenz und Sicherheit für die Patienten.

Es wird eine Grundstruktur offener Gruppen vorgestellt, die regelhaft aus der Begrüßung neuer Patienten, einem inhaltlichen Schwerpunkt sowie der Verabschiedung alter Patienten besteht. Der inhaltliche Schwerpunkt kann dabei 3 verschiedenen Durchführungsvarianten folgen: Er kann aus einem gemeinsam von allen bearbeiteten Thema bestehen, teilnehmerabhängig auf verschiedene Inhalte bzw. Patienten aufgeteilt werden oder eine Kombination dieser beiden Varianten darstellen. Ausschlaggebend für die Wahl der Durchführungsvariante sind die Fertigkeiten der einzelnen Gruppenteilnehmer (z.B. die Aufmerksamkeitsspanne) sowie die des Therapeuten, aber auch das jeweilige Thema.

In den Kapiteln 3 bis 13 werden schließlich für 11 verschiedene Themen- bzw. Störungsbereiche diverse, übersichtlich strukturierte und zum direkten Einsatz geeignete Arbeitsmaterialien präsentiert. Diese Themenbereiche sind: Alkoholmissbrauch, Alltagsplanung, Ärger und Aggression, Bewerbungstraining, Depression, Ernährung und Gesundheit, Essstörungen, Gehirnjogging, Schmerzbewältigung, Selbst- sicherheit und weibliche Identität. Jeder Abschnitt beginnt mit einer kurzen Information für den Therapeuten, in welcher der Hintergrund des Themas, Indikation, Schwerpunkte, benötigte Materialien, die empfohlene Durchführungsvariante sowie weitere Hinweise dargestellt werden. Ein ebenfalls für jedes Thema vorformulierter Begrüßungstext für Patienten ermöglicht einen ersten Einblick in das geplante Thema. Je nach Thema folgen dann zwischen 4 (Gehirnjogging) und 32 (Essstörungen) ABs. Zu Beginn eines Bausteins steht in der Regel ein AB, welches bereits vor Gruppenbeginn zur Vorbereitung mit dem Einzeltherapeuten bearbeitet werden sollte, beispielsweise die Analyse von Zielen oder eine exakte Beschreibung der Beschwerden. Es folgen Materialien zur Informationsvermittlung (z.B. Unterstützungsmöglichkeiten bei der Arbeitssuche, Depressionsmodell, Nährstoffe in Lebensmitteln, Schmerzwahrnehmung und -verarbeitung), zur Diagnostik und Analyse von Verhalten (z.B. Essprotokolle, individuelle Ärgergeschichte, Motive für die Lebensmittelwahl, Erfassung depressiver Kognitionen) sowie Interventionen bzw. deren Planung und Erfolgskontrolle (z.B. Ablehnungstraining, Anleitung für einen Wochenplan, Auszeit bei Ärger, Spiele für die Förderung der kognitiven Leistungsfähigkeit, Rollenspiele). Jedes Kapitel endet mit einem AB zu weiterführender Literatur. Zum Abschluss der Arbeitsmaterialien findet sich ein Dokumentationsbogen, in welchem der Therapeut die bearbeiteten Themen für jeden einzelnen Patienten kurz festhalten kann.

Zusammenfassend haben die Autoren mit «Offene Gruppen 1» ein vor allem für das (teil-)stationäre Setting sehr hilfreiches Arbeitsbuch vorgelegt, welches die flexible Vermittlung verschiedenster, äußerst relevanter Themen erleichtert. Ihrem Versprechen, diesem Buch weitere Ausgaben folgen zu lassen, haben die Autoren mit der Herausgabe von «Offene Gruppen 2» im Januar 2011 bereits Rechnung getragen.

Stephanie Körber, Erlangen

\section{Schramm, E.; Klecha, D. \\ Interpersonelle Psychotherapie in der Gruppe. \\ Das Kurzmanual \\ Stuttgart, Schattauer, 2010, 64 Seiten, 19,95 EUR \\ ISBN 3-7945-2758-5}

Interpersonelle Psychotherapie (IPT) ist eine in Deutschland für die Behandlung von affektiven Störungen und Essstörungen vom Wissenschaftlichen Beirat Psychotherapie

\section{KARGER}

Fax +497614520714

Information@Karger.de

www.karger.com
(C) 2011 S. Karger GmbH, Freiburg

ccessible online at:

www.karger.com/ver 
anerkannte Therapiemethode. Die IPT vermeidet, sich in ihrer Störungstheorie und Behandlungstheorie klar entweder einem lerntheoretischen oder einem psychodynamischen Konzept zuzuordnen, kann jedoch auch nicht problemlos als eigenständiges Verfahren betrachtet werden. IPT fokussiert in der Behandlung primär auf interpersonelle und im psychosozialen Kontext aktuell bedeutsame Themen und Problembereiche der Patienten. Diese sind: Einsamkeit/soziale Defizite, interpersonelle Konflikte, Rollenwechsel, Trauer und Verluste. Im Vergleich zur Einzeltherapie stehen bei der Gruppentherapieversion stärker strukturierende Maßnahmen, Psychoedukation und Übungen, auch in Form von Hausaufgaben, im Vordergrund. IPT arbeitet im Gegensatz zu anderen Psychotherapiemethoden bei Depression mit einem sehr medizinisch geprägten Modell, bei dem der Patient klar die Krankenrolle zugewiesen bekommt und Hoffnung sehr direktiv vermittelt wird: «Mit dem intensiven Therapieprogramm, das Ihnen hier angeboten wird und ganz speziell auf Depressionen zugeschnitten ist, müssten Sie sich in absehbarer Zeit wieder besser und leistungsfähiger fühlen» (Seite 9). In der praktischen Umsetzung wird eine große Zahl von Anleihen bei bekannten Verhaltenstherapiemethoden genommen, insbesondere bei der klassischen Verhaltenstherapie und der Dialektisch-Behavioralen Therapie. Es gibt eine sehr ausführliche Psychoedukation zum Schlaf inklusive Selbstbeobachtungsprotokollen, einer Liste angenehmer Aktivitäten, Wochenstrukturierungsplan, Emotionsmanagement, Stresstoleranzskills, Training interpersoneller Fertigkeiten und Problemlösetraining. Natürlich werden auch IPT-Kernthemen wie Rollenwechsel und Trauer behandelt. Bei dem Manual liegt die Betonung auf «kurz». Es handelt sich im Wesentlichen um eine ausführliche Inhaltsangabe der Gruppensitzungen. Insgesamt sind es 54 Seiten; hinzu kommen noch 68 Seiten Materialien, die man sich von der Verlagshomepage herunterladen muss. Allgemeine technische Aspekte der Umsetzung von IPT in Form einer Gruppentherapie und die Therapeutenrolle in der Gruppentherapie werden nur ganz kurz angerissen.

Insgesamt ein unverzichtbares Buch für Psychotherapeuten, die IPT in der Gruppenversion modellgetreu umsetzen wollen. Das Manual kann jedoch nur im Kontext weiterer Literatur und Ausbildung verstanden und umgesetzt werden. Kollegen, die sich allgemein über IPT informieren wollen oder Beratung zu typischen Schwierigkeiten in der Umsetzung von Gruppentherapie mit depressiven Patienten suchen, werden hier nicht ausreichend bedient.

Valerija Sipos, Lübeck
Bechdolf, A.; Pützfeld, V.; Güttgemanns, J.; Gross, S. Kognitive Verhaltenstherapie bei Personen mit erhöhtem Psychoserisiko. Ein Behandlungsmanual

Bern, Huber, 2010, 220 Seiten, 34,95 EUR

ISBN 978-3-456-84853-2

Seit einigen Jahren häufen sich Hinweise dafür, dass präventive Maßnahmen bei Personen mit erhöhtem Psychoserisiko die Entwicklung einer Schizophrenie zumindest deutlich nach hinten verzögern können. Daher ist nun auch die Zeit für ein deutschsprachiges Präventionsmanual, wie es von Bechdolf, Pützfeld, Güttgemanns und Gross vorgelegt wurde, reif.

Das Buch geht nach einer knappen Darlegung der gesundheitspolitischen Bedeutung der Prävention psychischer Störungen (Kapitel 1) zunächst auf die Geschichte des Schizophreniebegriffes ein (Kapitel 2). In Kapitel 3 werden die aus theoretischen Erwägungen hergeleiteten Ansatzpunkte für Prävention im Hinblick auf ihre Einsatzmöglichkeiten bei Schizophrenie diskutiert und Vor- und Nachteile medikamentöser versus psychologischer Therapie in diesem Stadium reflektiert. In Kapitel 4 erfolgt eine Darstellung des Forschungsstandes zur indizierten Prävention schizophrener Störungen, inklusive einer Darstellung bisheriger Therapiestudien. Den Schwerpunkt des Buches bildet dann ab Kapitel 5 die Darstellung der therapeutischen Techniken.

Das Buch ist im Aufbau überzeugend und didaktisch gut aufbereitet. Schön sind die zusammenfassenden Darstellungen in Form von gelungenen Abbildungen und Tabellen, die sich durch das Buch ziehen und sich positiv auf Lesbarkeit und Verständlichkeit auswirken. Anschaulich fand ich insbesondere die in Kapitel 4 aufgelisteten Kriterien zur Identifikation von Risikopersonen, aufschlussreich den Ansatz der Autoren, die deutschsprachige Tradition der Basissymptome mit dem von McGorry und Kollegen erstmals angewandten Konzept der «ultra-high risks» zusammenzuführen. In dem anschließenden Teil zu den Charakteristika von Personen mit erhöhtem Psychoserisiko hätte ich mir jedoch einen detaillierteren Einblick in die Grundlagenbefunde gewünscht. Dieser potenziell höchst therapierelevante Teil ist mit gerade eineinhalb Seiten doch sehr knapp ausgefallen. Hier listen die Autoren lediglich einige Befunde zu neuropsychologischen Einschränkungen bei Hochrisikoprobanden auf und bemängeln, dass es zu weiteren Variablenklassen des Vulnerabilitäts-StressBewältigungs-Modells kaum Befunde gäbe. Dabei vernachlässigen sie die umfangreiche Forschung zur Stresssensitivität bei Personen mit erhöhtem Psychoserisiko sowie die vielen therapierelevanten Befunde zu Selbstkonzepten und Verzerrungen der Informationsverarbeitung und der sozialen Kognition. Hier erweist es sich vielleicht als ungünstig, dass die Autoren im Einleitungsteil in Kapitel 2 auf ein recht altes Model von Nuechterlein und Dawson [1984] aufbauen und die aktuellen Weiterentwicklungen und Erweiterungen der VulnerabilitätsStress-Bewältigungs-Modelle um Komponenten der sozialen Kognition und kognitive Komponenten (z.B. neuere Weiter- 
entwicklungen der Gruppe um Nuechterlein oder Modelle der Gruppe um Garety) erst innerhalb der Anleitungen für die Therapie auftauchen. Dadurch kommt es zu einer mangelnden Passung zwischen der eher «old-school» neuropsychologisch basierten Grundlagenforschung und dem «new-school» kognitiven Vorgehen im Rahmen der Therapie.

Im Manualteil sind die dargestellten Interventionen klar und verständlich geschildert, und die praktische Anwendung wird durch die vielen Arbeitsblätter weiter erleichtert. Auffallend ist, dass die Interventionen fast deckungsgleich sind mit denen, die in den «Cognitive Behavioral Therapy»-Manualen für die Behandlung schizophrener Positivsymptomatik zu finden sind. Dies spiegelt aber wohl den Stand der Präventionsforschung im Bereich der Schizophrenie wider, die im Moment noch wenig spezifische Beiträge zur Prävention zu verzeichnen hat. Ein paar spezifische Hinweise zur «Prävention» enthält der Therapieteil dennoch, etwa wenn es darum geht, wie man Betroffenen erklärt, dass sie unter einem erhöhten Psychoserisiko leiden, oder warum man lieber von psychotischer Krise sprechen und den Begriff Schizophrenie vermeiden sollte.

Ein richtiger Wermutstropfen ist das Titelbild, das vom Verlag ausgewählt wurde. Schade, dass bei Büchern zur Schizophrenie immer wieder auf einen gespaltenen Geist angespielt werden muss, wodurch das sich hartnäckig haltende Stereotyp der gespaltenen Persönlichkeit weiter bedient wird. Vom Kauf des insgesamt gelungenen Buches möchte ich mit dieser Kritik nicht abraten, aber man kann es ja vielleicht einfach andersherum hinlegen.

Tania Lincoln, Hamburg

Görtz-Dorten, A.; Döpfner, $M$.

\section{Therapieprogramm für Kinder mit aggressivem Verhalten (THAV)}

Göttingen, Hogrefe, 2010, 182 Seiten, 59,95 EUR, mit CD-ROM

ISBN 978-3-8017-2084-1

«Aggressives Verhalten von Kindern tritt häufig im Kontext aggressiv-dissozialer Verhaltensweisen auf. Die Therapie dieser Probleme stellt eine besondere Herausforderung dar, weil diese Störungen häufig auftreten, oft einen chronischen Verlauf haben und insgesamt schwer zu behandeln sind» (Zitat aus Kapitel 1). Das Therapieprogramm für Kinder mit aggressivem Verhalten (THAV), konzipiert auf Grundlage der multimodalen Kinder- und Jugendlichenpsychotherapie, wurde für die Behandlung von Kindern mit aggressiven Verhaltensauffälligkeiten Gleichaltrigen gegenüber im Alter von 6 bis 12 Jahren entwickelt. Es beinhaltet Module mit patientensowie umfeldzentrierten Interventionen, die sowohl in der Einzel- wie auch Gruppentherapie Verwendung finden können. Die Module und Interventionen können unter Berück- sichtigung aufrechterhaltender Faktoren und daraus abgeleiteter Interventionsbausteine individuell zusammengestellt werden. Ergänzt werden die kindzentrierten Interventionen durch familien- und schulzentrierte Verfahren zur Veränderung dysfunktionaler Kognitionen und Erwartungen bei den Eltern/Bezugspersonen, zum Abbau aggressionsverstärkenden Erziehungsverhaltens und zur Einführung von Belohnungen für sozial kompetentes Verhalten des Kindes. Das Therapieprogramm wird ergänzt durch umfangreiches Arbeitsmaterial auf einer mitgelieferten CD-ROM und eine Materialbox, die zusätzlich erworben werden kann.

Das Buch gliedert sich in 2 Kapitel. Kapitel 1 (21 Seiten) gibt einen Überblick über Symptomatik und Häufigkeit aggressiven Verhaltens, Komorbidität und Verlauf, mögliche Ursachen und therapeutische Ansatzpunkte. Die Hilfe zur erfolgreichen Problembewältigung bildet den Schwerpunkt der Therapie, ergänzt durch Strategien zur Ressourcenaktivierung, Problemfokussierung und kognitiv-affektiven Klärung. Um die Wirksamkeit der Therapie zu steigern und die Übertragung in den Alltag zu verbessern, werden begleitend von den Autoren familien-, schul- und gleichaltrigenzentrierte Interventionen vorgeschlagen. Weiter gehen die Autoren auf bisher vorliegende Therapieansätze und deren Wirksamkeit ein und beschreiben Ergebnisse einer Pilotstudie sowie Ziele einer laufenden Hauptstudie zur Wirksamkeit des THAV.

In Kapitel 2 (144 Seiten) geben die Autoren zunächst einen Überblick über die Indikation für das Therapieprogramm. Es werden mögliche psychosoziale Merkmale, die mit der Entwicklung aggressiven Verhaltens in Verbindung gebracht werden - die Tendenz zur Fehlwahrnehmung und Fehlinterpretation sozialer Situationen, beeinträchtigte Empathie, Defizite in der moralischen Entwicklung, mangelnde soziale Problemlösefähigkeit, soziale Kompetenzdefizite, verminderte Affekt- und Impulskontrolle und beeinträchtigte Beziehungen zu Gleichaltrigen - erläutert. Darauf aufbauend werden die therapeutischen Ansatzpunkte für die verschiedenen Module und Therapiebausteine erklärt.

Das THAV besteht aus 5 Modulen und einem modulübergreifenden Spiel. Entsprechend den individuellen Problemen der Kinder können die Module und Bausteine anhand eines Entscheidungsbaums ausgewählt und die Therapie geplant werden. Die Module beinhalten Hinweise zu Therapievorbereitung, Diagnostik und Verlaufskontrolle (Modul I), sozialkognitive Interventionen zur Verminderung von Störungen der Informationsverarbeitung (Modul II), ein Ärgerkontrolltraining zur Entwicklung und Stärkung der Impulskontrolle (Modul III), ein Problemlöse- und Fertigkeitentraining sowie Interventionen zur Modifikation sozialer Interaktionen (Modul IV) und Hinweise zum Therapieabschluss mit Rückfallprävention und Ablösung (Modul V). Es werden bei den einzelnen Interventionsbausteinen Vorschläge für den Ablauf der einzelnen Sitzungen gemacht, eine genaue zeitliche Sitzungsstruktur vorgeschlagen und Hinweise zum Umgang mit schwierigen Therapiesituationen gegeben. 
Die Autoren geben an, das Therapieprogramm sei für den Altersbereich von 6 bis 12 Jahren geeignet. Die teils eher «jugendspezifischen» Formulierungen auf den Arbeitsblättern und manche (Selbstbeobachtungs-)Übungen dürften jedoch für jüngere Kinder noch schwer verständlich bzw. umsetzbar sein. Zusammenfassend handelt es sich beim THAV aber um ein anschauliches und gut strukturiertes Therapiemanual mit zahlreichen Materialien und Anregungen. Es enthält alle wesentlichen Informationen zum Störungsbild sowie zahlreiche Interventionsvorschläge, abgerundet durch konkrete Durchführungshinweise und Formulierungshilfen.

Claudia Trebes, Erlangen

Klos, H.; Görgen, W.

Rückfallprophylaxe bei Drogenabhängigkeit. Ein Trainingsprogramm

Göttingen, Hogrefe, 2009, 158 Seiten, 44,95 EUR

ISBN 978-3-8017-2174-9

Das Rückfallprophylaxetraining (RPT) für drogenabhängige Menschen setzt sich aus 16 Modulen zusammen. Jedes Modul umfasst 90 min und hat eine regelmäßige, in sich geschlossene Struktur. Die Module bauen aufeinander auf. In den Modulen 1-3 werden Grundinformationen vermittelt. In Modul 1 werden Informationen über Rückfallwahrscheinlichkeit, Rückfallzeitpunkt und Rückfallgeschehen an die Patienten weitergegeben, in Modul 2 werden die Phasen der Veränderung nach dem Transtheoretischen Modell von Prochaska und DiClemente veranschaulicht und in Modul 3 werden individuelle Schutzfaktoren entwickelt. In den Modulen 4-7 werden Rückfallursachen und Rückfallvermeidung bearbeitet. Die Auseinandersetzung hierfür erfolgt über das Erkennen von Risikofaktoren (Modul 4), das Benennen der Ambivalenzen der Drogenfreiheit (Modul 5) sowie über Strategien im Umgang mit Drogenverlangen (Modul 6) und über Strategien in rückfallrelevanten Situationen (Modul 7). In den Modulen 8-11 werden spezifische Themen wie Drogenabhängigkeit und Alkoholkonsum (Modul 8), Kriminalität und Rückfälligkeit (Modul 9), Kriminalität und materielle Sicherheit (Modul 10) sowie Risikobereitschaft und Rückfallgeschehen (Modul 11) aufgegriffen. In Modul 12 geht es um Rückfallmanagement, d.h. was muss nach einem Rückfall passieren, um die Abstinenz wiederherzustellen. In den Modulen 13-15 stehen Themen zur Ressourcenorientierung im Vordergrund, wie Angehörige und Rückfallgeschehen (Modul 13), Lust und andere gute Gefühle (Modul 14) sowie Erfolge, Anerkennung und Belohnung (Modul 15). Den Abschluss bildet das Modul 16, das der Wiederholung und der Bilanz dient.

Das Buch von Hartmut Klos und Wilfried Görgen ist ein anschauliches Rückfallprophylaxemanual für drogenabhängige Patienten. Der große Vorteil dieses Buches besteht vor allem in der handlungsorientierten und praxiserprobten Herangehensweise. Die Gestaltung der Arbeitsblätter ist klar und übersichtlich, auch die Arbeitsanweisungen für die einzelnen Module sind sehr strukturiert. Die Autoren benutzen eine Vielzahl von Methoden aus der humanistischen Psychologie, wie z.B. das soziale Atom, und schaffen hierüber erlebnisorientierte Lernformen. Sie benutzen dabei viele Bilder aus dem Alltag als Metaphern, wie das des Airbag-Modells, die anschaulich und patientennah sind. Ein deutlicher Nachteil dieses Manuals ist jedoch, dass es bisher noch nicht wissenschaftlich evaluiert ist und damit noch nicht in seiner Wirksamkeit bestätigt wurde. Auch fehlen inhaltlich noch konkrete Strategien zur Emotionsregulation; diese wären gerade für Patienten, die zusätzlich an einer Persönlichkeitsstörung oder an einer posttraumatischen Belastungsstörung leiden, sehr hilfreich und notwendig. Insgesamt ist dieses Gruppenmanual ein sehr gutes Basiswerk, um Drogenpatienten in ihrer Abstinenz zu stärken und um sie im Umgang mit Risikosituationen zu trainieren.

Nadja Tahmassebi, Friedrichsdorf 